\title{
Effect of the anti-IL-17 antibody on allergic inflammation in an obesity-related asthma model
}

\author{
Lin Liang, Jung Hur, Ji Young Kang, Chin Kook Rhee, Young Kyoon Kim, and Sook Young Lee
}

Division of Pulmonary, Allergy and Critical Care Medicine, Department of Internal Medicine, College of Medicine, Seoul St. Mary's Hospital, The Catholic University of Korea, Seoul, Korea

Received: June 8, 2017

Revised : August 2, 2017

Accepted: September 29, 2017

\section{Correspondence to}

Sook Young Lee, M.D.

Division of Pulmonary, Allergy and Critical Care Medicine, Department of Internal Medicine, College of Medicine, Seoul St. Mary's Hospital, The Catholic University of Korea, 222 Banpo-daero, Seocho-gu, Seoul 06591, Korea

Tel: +82-2-2258-6061

Fax: $+82-2-2258-1219$

E-mail: sooklee@catholic.ac.kr
Background/Aims: The co-occurrence of obesity aggravates asthma symptoms. Diet-induced obesity increases helper $\mathrm{T}$ cell (TH) 17 cell differentiation in adipose tissue and the spleen. The 3-hydroxy-3-methylglutaryl-coenzyme A reductase inhibitor pravastatin can potentially be used to treat asthma in obese patients by inhibiting interleukin 17 (IL-17) expression. This study investigated the combined effects of pravastatin and anti-IL-17 antibody treatment on allergic inflammation in a mouse model of obesity-related asthma.

Methods: High-fat diet (HFD)-induced obesity was induced in C57BL/6 mice with or without ovalbumin (OVA) sensitization and challenge. Mice were administered the anti-IL-17 antibody, pravastatin, or both, and pathophysiological and immunological responses were analyzed.

Results: HFD exacerbated allergic airway inflammation in the bronchoalveolar lavage fluid of HFD-OVA mice as compared to OVA mice. Blockading of the IL-17 in the HFD-OVA mice decreased airway hyper-responsiveness (AHR) and airway inflammation compared to the HFD-OVA mice. Moreover, the administration of the anti-IL-17 antibody decreased the leptin/adiponectin ratio in the HFD-OVA but not the OVA mice. Co-administration of pravastatin and anti-IL-17 inhibited airway inflammation and AHR, decreased goblet cell numbers, and increased adipokine levels in obese asthmatic mice.

Conclusions: These results suggest that the IL-17-leptin/adiponectin axis plays a key role in airway inflammation in obesity-related asthma. Our findings suggest a potential new treatment for IL-17 as a target that may benefit obesity-related asthma patients who respond poorly to typical asthma medications.

Keywords: Asthma; Obesity; Anti-interleukin-7; Pravastatin; Inflammation

\section{INTRODUCTION}

Obesity is associated with a chronic inflammatory response, including abnormal adipokine secretion and activation of various proinflammatory signaling pathways [1]. These inflammatory processes have a risk factor for obesity and its comorbidities, such as insulin resistance, type 2 diabetes, and cardiovascular diseases [1].

Asthma is a chronic inflammatory condition char- acterized by airway hyper-responsiveness (AHR) and reversible airflow obstruction [2]. Recent evidence suggests that obesity is a risk factor for asthma [3] since it heightens inflammation in the lungs and thereby decreases pulmonary functional volume [4]. In addition, airway caliber is reduced as body mass index increases [5]. Some animal studies have shown that obesity aggravates preexisting asthma by enhancing eosinophilic inflammation and AHR independent of ovalbumin (OVA) 
sensitization in a mouse model $[6,7]$. Asthma patients who are obese respond poorly to typical asthma medications such as corticosteroids [8]. Weight loss and bariatric surgery do not always improve AHR in obese asthmatic patients with allergic (helper T cell $\left[\mathrm{T}_{\mathrm{H}}\right]$ 2) airway inflammation [9]. Hence, asthma accompanied by both an allergic phenotype and obesity might be associated with extra complications.

Diet-induced obesity increases $\mathrm{T}_{\mathrm{H}} 17$ differentiation in adipose tissue and the spleen [10]. Interleukin 17 (IL-17) released by $\mathrm{T}_{\mathrm{H}^{17}}$ cells in the spleen stimulates innate lymphoid cell 3 (ILC-3) in the lungs in obese individuals [10]. Obesity is also associated with increased levels of proinflammatory cytokines in adipose tissue, including tumor necrosis factor- $\alpha$, IL- 6 , and IL-1 $\beta$ [11]. IL-1 $\beta$ produces IL-17 release from ILC-3, promoting airway reactivity in obesity-related asthma [12]. Moreover, IL-17 can directly cause AHR by acting on airway smooth muscle cells and inducing neutrophil inflammation in severe asthma $[13,14]$. As a common factor in both asthma and obesity, IL-17 might be an appropriate therapeutic target for the treatment of obesity-related asthma.

Statins inhibit 3-hydroxy-3-methylglutaryl-coenzyme A reeducates to suppress cholesterol biosynthesis and are primarily used for the treatment of obesity-related dyslipidemia and the prevention of cardiovascular disease [15]. It has been suggested that statins can be used to treat autoimmune disease based on the finding that these drugs inhibit $\mathrm{T}_{\mathrm{H}^{17}}$ differentiation [16]. Specifically, statins alter the $\mathrm{T}_{\mathrm{H}} 1 / \mathrm{T}_{\mathrm{H}^{2}}$ balance and decrease IL-17 levels $[17,18]$. Animal studies have shown that pravastatin suppresses OVA-induced airway inflammation by reducing $\mathrm{T}_{\mathrm{H}^{2}}$ cytokine levels and IL-17 production [18]. These observations suggest that attenuating systemic inflammation with statins could benefit obese asthma patients.

To test this hypothesis, the present study investigated the effects of treatment with pravastatin and an anti-IL$17 \mathrm{~A}$ antibody in a mouse model of obesity-related asthma.

\section{METHODS}

\section{Mice and diet}

Female $\mathrm{C}_{57} \mathrm{BL} / 6$ mice (4 weeks old; Orient Bio, Seongnam, Korea) were used for experiments. Mice were fed standard chow (2018, Harlan Laboratories, Indianapolis, IN, USA) or were placed on a high-fat diet (HFD) (D12492, Harlan Laboratories), in which fat accounted for $60 \%$ of the total calories, for 12 consecutive weeks. Body weight was recorded weekly. Animal experiments were approved by the Institutional Animal Care and Use Committees of the Catholic University of Korea (approval no. CUMC-2016-0117-02).

\section{Sensitization and antigen challenge}

Lean and obese mice were immunized at weeks 9 and 10 by subcutaneous injection of $25 \mu \mathrm{g}$ OVA grade $\mathrm{V}$ (Sigma-Aldrich, St. Louis, MO, USA) adsorbed to $1 \mathrm{mg}$ aluminum hydroxide (Aldrich, Milwaukee, WI, USA) in $200 \mu \mathrm{L}$ normal saline. Two weeks later (i.e., at week 12), OVA (20 $\mu \mathrm{g} / 50 \mu \mathrm{L}$ in phosphate-buffered saline [PBS]) was intranasally administered 3 consecutive times under isoflurane anesthesia (Vedco, St. Joseph, MO, USA). Ageand sex-matched control mice were administered PBS in the same manner. Mice were sacrificed 24 hours after the final OVA challenge, and bronchoalveolar lavage fluid (BALF) and lung tissue were collected.

\section{Anti-IL-17 antibody and pravastatin treatment}

Mice were administered pravastatin (Cayman, Ann Arbor, MI, USA; $10 \mathrm{mg} / \mathrm{kg}$ ) by intraperitoneal injection twice during weeks 9 and 10 and daily during week 12. To neutralize IL-17A, mice were treated with the anti-mouse IL-17A monoclonal antibody (R\&D Systems, Minneapolis, MN, USA; $50 \mu \mathrm{g}$ ) by intraperitoneal injection twice during weeks 9 and 10 and three times during week 12. Mice were sacrificed after the last OVA challenge and administration of the final dose of pravastatin/anti-IL-17 antibody.

\section{Measurement of AHR}

The FlexiVent system was used to assess AHR (SCIREQ, Montreal, QC, Canada). To determine baseline airway respiratory resistance $(\mathrm{Rrs})$ values, mice were exposed to nebulized PBS for 3 minutes, followed by progressive exposure to $6.25,12.5,25$, and $50 \mathrm{mg} / \mathrm{mL}$ nebulized methacholine (Sigma-Aldrich) using an aerosonic ultrasonic nebulizer (DeVilbiss, Somerset, PA, USA). Each test lasted for 3 minutes, and average Rrs values were calculated to determine methacholine concentration.

\section{BALF}

Prior to thoracotomy, mice were anesthetized by intra- 
peritoneal injection. A silicone tube connected to an $800-\mu \mathrm{L}$ tuberculin injector with a 23-gauge needle was inserted into the trachea, and $1.2 \mathrm{~mL}$ sterile PBS was delivered into the right lung. The recovered BALF was centrifuged for 7 minutes at $353 \times g$ and $4^{\circ} \mathrm{C}$, and the supernatant was stored at $-70^{\circ} \mathrm{C}$. A LUNA automated cell counter (Logos Biosystems, Annandale, VA, USA) was used to determine total cell number in the BALF. After dilution to $1 \times 10^{6}$ cells $/ \mathrm{mL}, 50-\mu \mathrm{L}$ aliquots of BALF were transferred to microscope slides and centrifuged for 5 minutes at $43 \times g$ and stained with Diff-Quick (Sysmax, Kobe, Japan). The percentages of macrophages, eosinophils, lymphocytes, and neutrophils in the BALF were determined by counting 500 leukocytes in randomly selected fields under a light microscope.

\section{Enzyme-linked immunosorbent assay}

IL-4, IL-5, IL-13, and IL-17A, adiponectin, and leptin concentrations in the BALF were estimated by enzyme-linked immunosorbent assay (ELISA) using commercial kits (R\&D Systems).

\section{Histopathology}

The left lung was filled with $4 \%$ paraformaldehyde solution and embedded in paraffin. Lung sections were stained with hematoxylin and eosin (H\&E) to assess tissue inflammation, and with periodic acid-Schiff(PAS) to identify goblet cell hyperplasia and submucosal gland hypertrophy.

\section{Statistical analysis}

Data were analyzed using Prism software (GraphPad Inc., San Diego, CA, USA). Changes in AHR and body weight were evaluated by two-way analysis of variance (ANOVA) and a Bonferroni post hoc test, whereas differences between other variables were compared by oneway ANOVA and a Bonferroni post hoc test. A $p<0.05$ was considered statistically significant.

\section{RESULTS}

\section{HFD induces obesity and changes in adipokine levels in an asthma model}

Wild-type mice were fed HFD for 12 weeks, starting at 4 weeks of age. The gross appearance and volume of ad- ipose tissue differed between the HFD-OVA and standard-diet groups, although the lung tissue was similar (Fig. 1A). HFD-OVA induced significant weight gain as compared to mice fed standard chow (Fig. 1B). We compared adipokine levels in obese and non-obese asthmatic mice and found that animals in the HFD-OVA group had higher leptin levels than controls. Leptin levels differed significantly between the HFD-OVA and OVA groups, whereas adiponectin levels were reduced in the HFD and HFD-OVA groups, with lower levels in the HFD-OVA than in the OVA group. Accordingly, the leptin/adiponectin ratio was higher in the HFD-OVA than in the OVA and control groups (Fig. 1C). These results confirm that HFD caused obesity in a mouse model of asthma induced by OVA. Moreover, HFD-induced obesity was associated with increased leptin levels in asthmatic mice.

\section{Obesity causes pathophysiological changes in an asthma model}

To determine whether obesity exacerbates asthma, mice in the HFD-OVA group were subjected to OVA sensitization. Mice treated with OVA and fed standard chow or HFD showed resistance to methacholine but did not exhibit any obvious differences in AHR. However, mice in the HFD group showed slightly increased AHR relative to controls (Fig. 2A). We also found that total cell counts, as well as the number of macrophages, eosinophils, neutrophils, and lymphocytes, in the BALF were elevated in the OVA and HFD-OVA groups. Interestingly, total cell number and macrophage and eosinophil counts in the BALF were higher in the HFD-OVA than in the OVA group. In contrast, no differences were observed between the HFD and control groups (Fig. 2B).

A histopathological analysis of lung tissue revealed that compared to control animals, eosinophilic inflammation was increased in the OVA and HFD-OVA groups, with a higher degree of inflammation detected in the latter by H\&E staining. There was no eosinophilic inflammation in HFD mice relative to controls (Fig. 2C).

Pathological changes in the lungs of asthmatic animals were investigated by evaluating goblet cell hyperplasia in the peribronchiolar area. Hyperplasia was detected in OVA and HFD-OVA mice as compared to the control, with no difference between the former two groups. Moreover, HFD did not induce goblet cell 


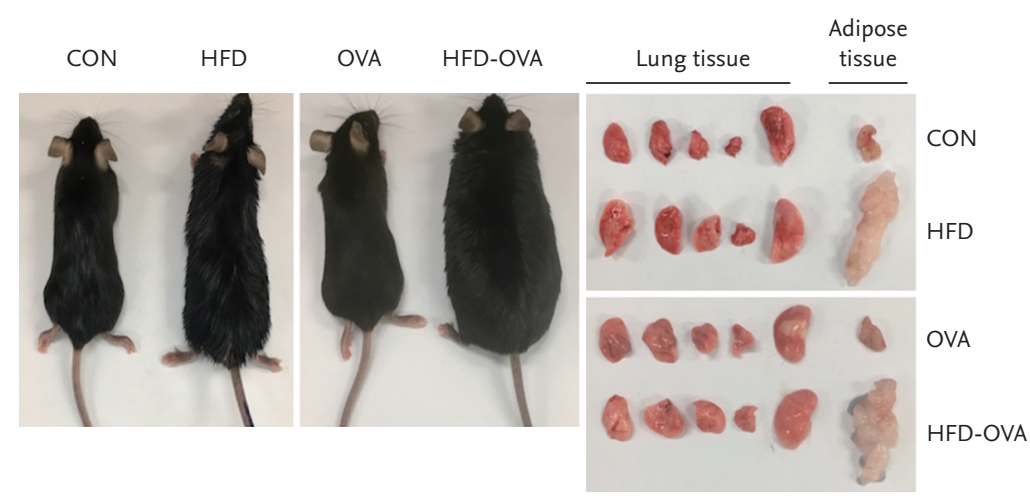

A

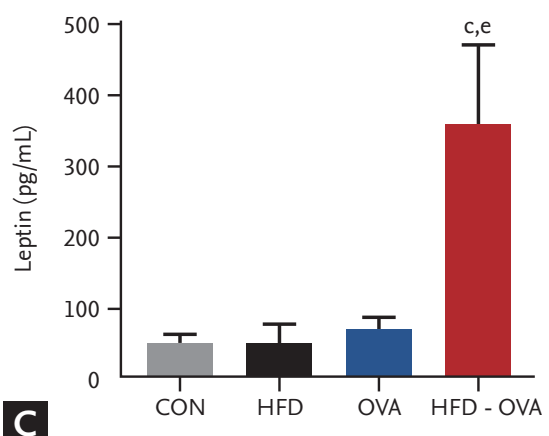

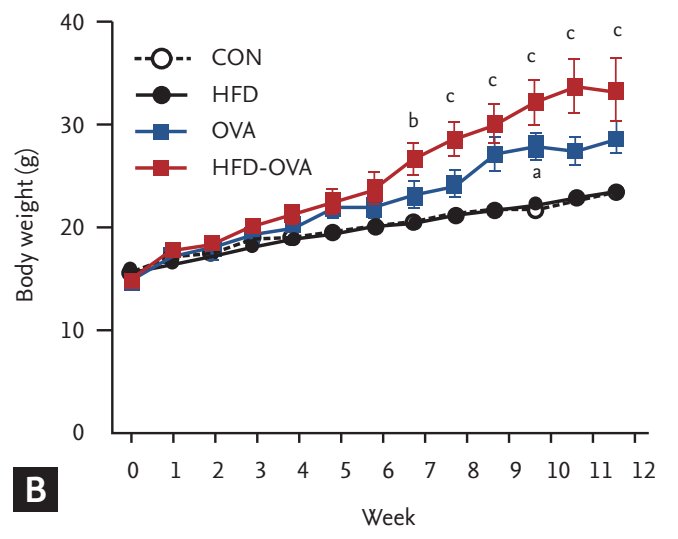
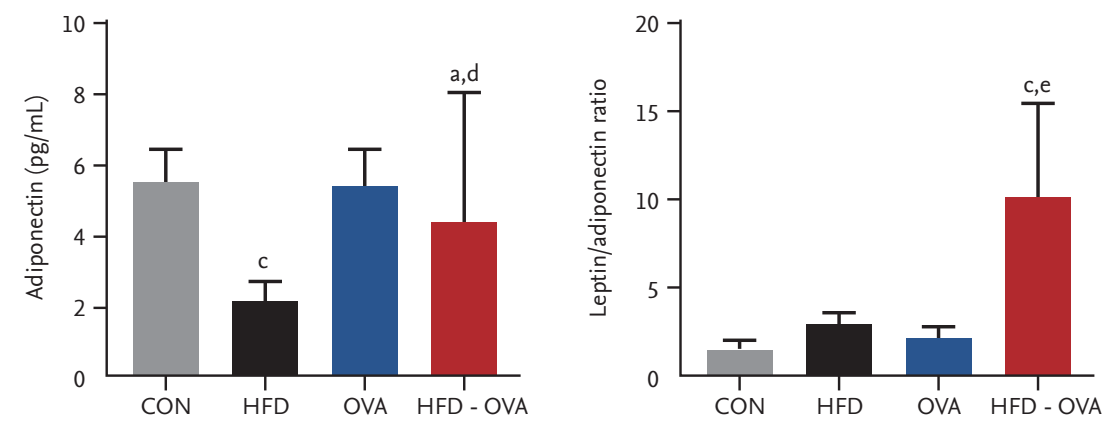

Figure 1. High-fat diet (HFD)-induced obesity and changes in adipokine levels in a mouse model of asthma. Mice were fed standard chow or HFD for 12 weeks. (A) Representative images of mice and organs. (B) Body weight in mice on a normal diet or HFD. (C) Leptin and adiponectin levels and leptin/adiponectin ratio in the bronchoalveolar lavage fluid as determined by enzyme-linked immunosorbent assay. Results are expressed as mean \pm SEM ( $n=5$ to 7 per group). CON, control; OVA, ovalbumin. ${ }^{a} p<0.05,{ }^{b} p<0.01,{ }^{c} p<0.001$ vs. CON group, and ${ }^{d} p<0.05,{ }^{e} p<0.001$ vs. OVA group.

proliferation, as determined by PAS staining (Fig. 2C). Collectively, obese asthmatic animals showed the distinctive asthmatic features of airway inflammation unlike non-obese asthmatic animals, whereas there was no difference between HFD and control mice. Thus, we asked what factors worsen the inflammatory response in obesity-related asthma?

\section{Obesity exacerbates airway inflammation by in- creasing $T_{H^{2}}$ and $T_{H^{17}}$ cytokine levels}

Previous studies have shown that the level of the proinflammatory cytokine IL-17 was elevated in obesity-related asthma, causing AHR by directly acting on airway smooth muscle cells [19]. We hypothesized that IL-17 induced airway inflammation in obese asthmatic mice. Notably, levels of $\mathrm{T}_{\mathrm{H}^{2}}$ and $\mathrm{T}_{\mathrm{H}} 17$ cytokines (including IL4, IL-5, IL-13, and IL-17) were increased in the OVA and HFD-OVA groups (Fig. 3). Moreover, IL-4, IL-5, and IL-17 levels were higher in the latter than in the former (Fig. ${ }_{3} \mathrm{~A},{ }_{3} \mathrm{~B}$, and $\left.{ }_{3} \mathrm{D}\right)$. However, there were no differences in IL-13 levels between the two groups (Fig. ${ }_{3} \mathrm{C}$ ). In contrast, IL-4, IL-5, IL-13, and IL-17 levels in the HFD group were similar to those in control mice (Fig. 3).

\section{Anti-IL-17 antibody treatment alleviates AHR and airway inflammation in obesity-related asthma}

$\mathrm{T}_{\mathrm{H}^{17}}$-mediated immunity is critical for inflammation in adipose tissue associated with obesity [20]. To investigate the role of $\mathrm{IL}-17$ in obesity-related asthma, the protein was blocked by the administration of the anti-IL-17 antibody before the first OVA sensitization. This resulted in AHR in the OVA and HFD-OVA groups, as evidenced by a decrease in the Rrs value relative to untreated obese and non-obese mice with asthma (Fig. 4A). In addition, IL-17 blockade suppressed inflammatory cell infiltration, including total cells, macrophages, and 


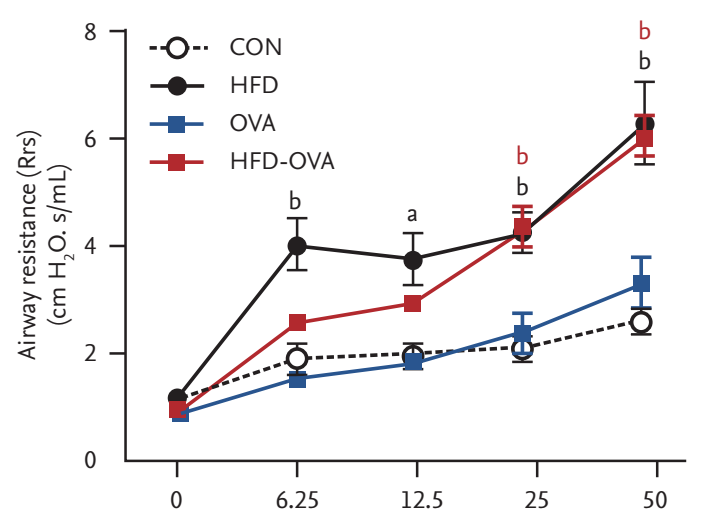

A

Methacholine $(\mathrm{mg} / \mathrm{mL})$

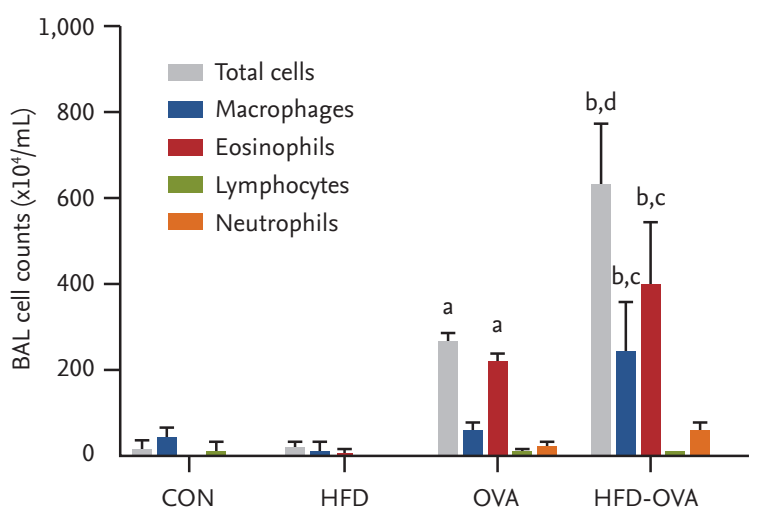

B

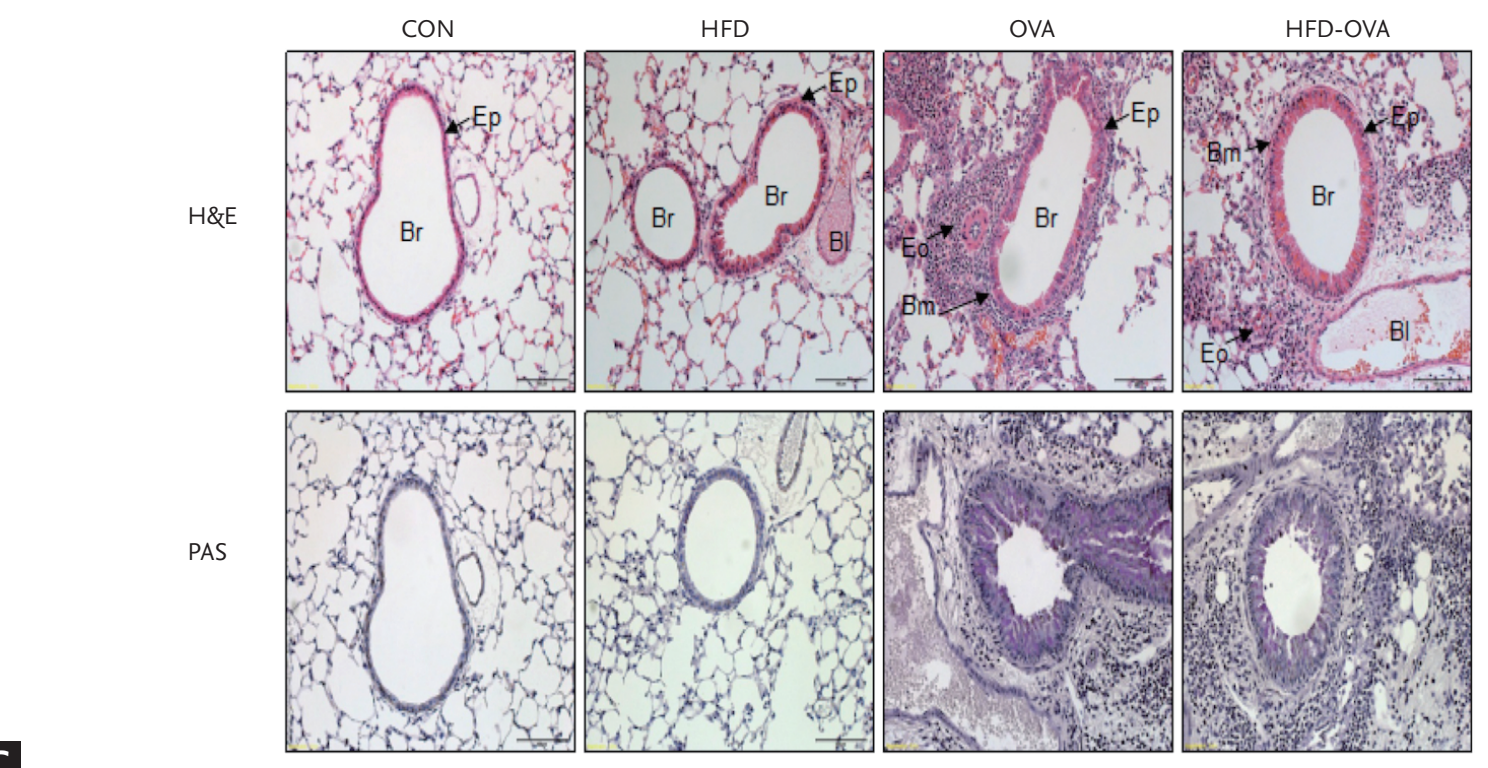

C

Figure 2. Co-occurrence of obesity causes pathophysiological changes in an asthma model. (A) Airway hyper-responsiveness was measured as respiratory resistance 24 hours after the final ovalbumin (OVA) challenge. (B) Effect of an high-fat diet (HFD) on cell counts in the bronchoalveolar lavage fluid (BALF). Mice were sacrificed 24 hours after the final OVA challenge, and BALF cells were isolated. (C) Paraffin-embedded lung tissue sections were stained with H\&E and periodic acid-Schiff (PAS) ( $\times 200$ ). Results are expressed as mean \pm SEM ( $n=5$ to 7 per group). CON, control; Br, bronchus; Bm, basement membrane; Eo, eosinophil; Ep, epithelium; Bl, blood vessel. ${ }^{a} p<0.01,{ }^{b} p<0.001$ vs. control group, and ${ }^{c} p<0.01,{ }^{d} p<0.001$ vs. OVA group.

eosinophils, in the HFD-OVA group (Fig. 4B). In contrast, anti-IL-17 antibody treatment increased total cell and eosinophil counts in the OVA group. A microscopic examination of lung tissue revealed that IL-17 blockade decreased and increased inflammatory cell infiltration in the HFD-OVA and OVA groups, respectively, as determined by H\&E staining (Fig. $4 \mathrm{C}$ ) and decreased the number of goblet cells in both groups, as determined by PAS staining. These data suggest that IL-17 reduces pulmonary function by exacerbating airway inflammation in obese asthmatic mice.

\section{IL-17 blockade suppresses $T_{H^{2}}$ cytokines and im- proves adipokine levels in obesity-related asthma}

Given the observation that IL-17 positively regulates airway inflammation in HFD-OVA mice, we examined the effect of IL-17 neutralization on the levels of $\mathrm{T}_{\mathrm{H}^{2}}$-type cytokines and adipokines. IL-17 blockade decreased and increased IL-4, IL-5, and IL-13 levels in the HFD-OVA and OVA groups, respectively (Fig. 5A-5C). The leptin 
A
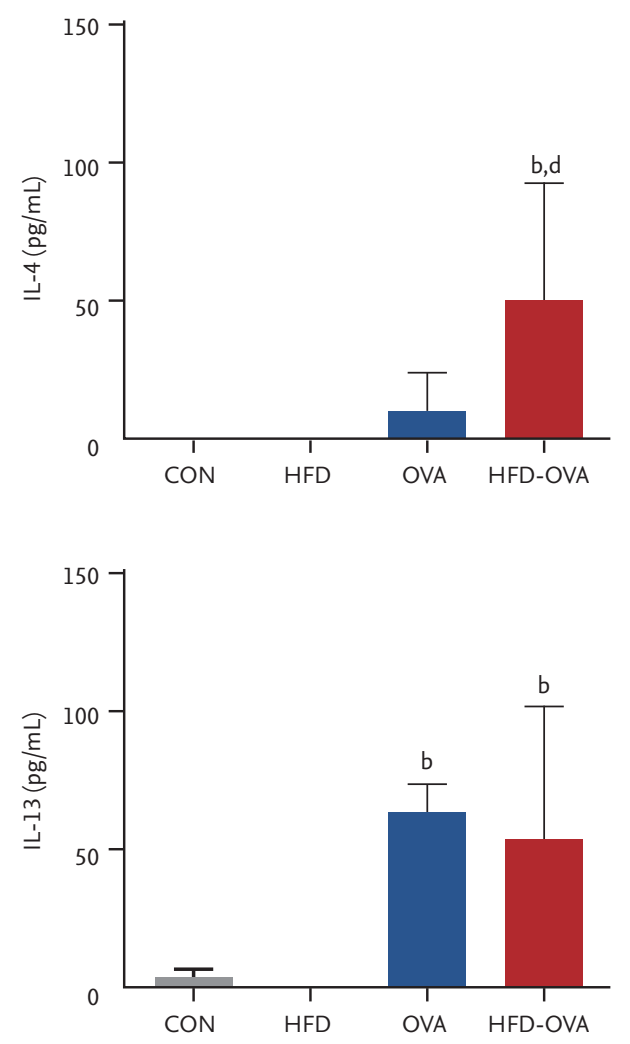

B

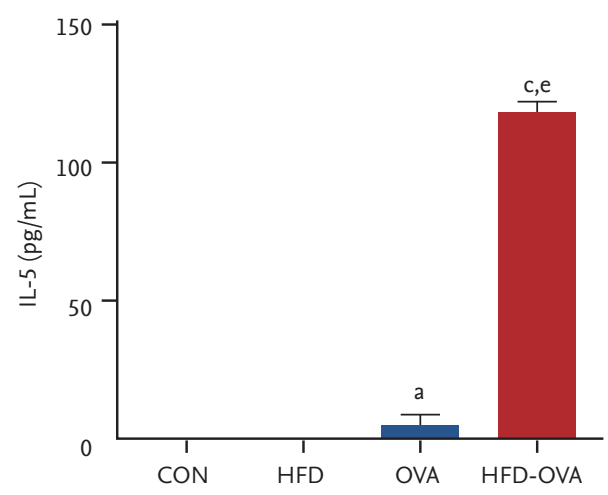

D

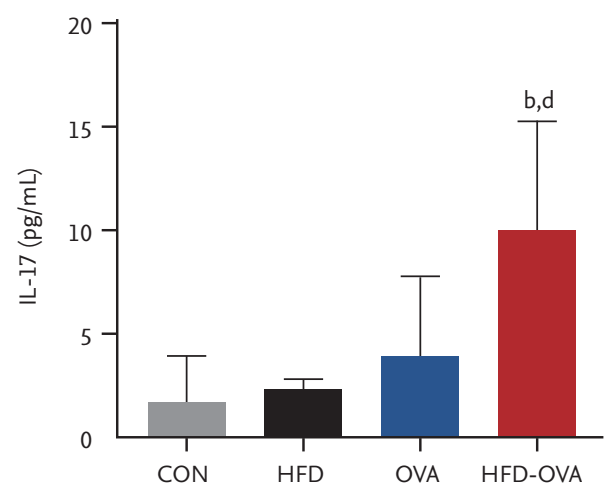

Figure 3. Obesity exacerbates airway inflammation by increasing $\mathrm{T}_{\mathrm{H}}$ and $\mathrm{T}_{\mathrm{H}} 17$ cytokine levels. (A) Interleukin 4 (IL-4), (B) IL5, (C) IL-13, and (D) IL-17 levels in the bronchoalveolar lavage fluid were evaluated by enzyme-linked immunosorbent assay. Results are expressed as mean $\pm \operatorname{SEM}\left(\mathrm{n}=5\right.$ to 7 per group). CON, control; HFD, high-fat diet; OVA, ovalbumin. ${ }^{\mathrm{a}} \mathrm{p}<0.05,{ }^{\mathrm{b}} \mathrm{p}<0.01$, ${ }^{c} p<0.001$ vs. control group, and ${ }^{d} p<0.05,{ }^{e} p<0.001$ vs. OVA group.

level increased in HFD-OVA as compared to control mice; this effect was partly reversed by anti-IL-17 antibody treatment. There were no differences in leptin levels between the OVA and OVA + anti-IL-17 groups (Fig. $\left.{ }_{5} \mathrm{D}\right)$. IL-17 blockade increased adiponectin levels in the HFD-OVA group but had no effect in the OVA group (Fig. 5E). HFD-OVA mice had a higher mean leptin/ adiponectin ratio than OVA and control mice, with no difference observed between the latter two groups. Administration of the anti-IL-17 antibody decreased the ratio in the HFD-OVA but not the OVA group (Fig. $5 \mathrm{~F}$ ). These data revealed that IL-17 plays a regulatory role of leptin/adiponectin ratio in obese asthma. Furthermore, its regulatory functions exacerbate uncontrolled asthmatic features in obese asthma patients compared with non-obese asthma patients.
Pravastatin and anti-IL-17 antibody treatment improves AHR and airway inflammation in obesity-related asthma

We next investigated whether pravastatin combined with anti-IL-17 antibody treatment could alleviate asthma symptoms in obese mice. The increase in Rrs values in the HFD-OVA group relative to controls was mitigated by IL-17 blockade and was further reduced by treatment with both pravastatin and the anti-IL-17 antibody (Fig. 6A). The combination of pravastatin and anti-IL-17 antibody also reduced total cell and alveolar eosinophil and macrophage numbers in the BALF of HFD-OVA mice. However, no differences were observed between HFD-OVA + anti-IL-17 antibody treatment groups with and without pravastatin treatment (Fig. 6B).

Microscopic examination of mouse lung tissue revealed that combined treatment with pravastatin and anti-IL-17 antibody decreased inflammatory cell infil- 

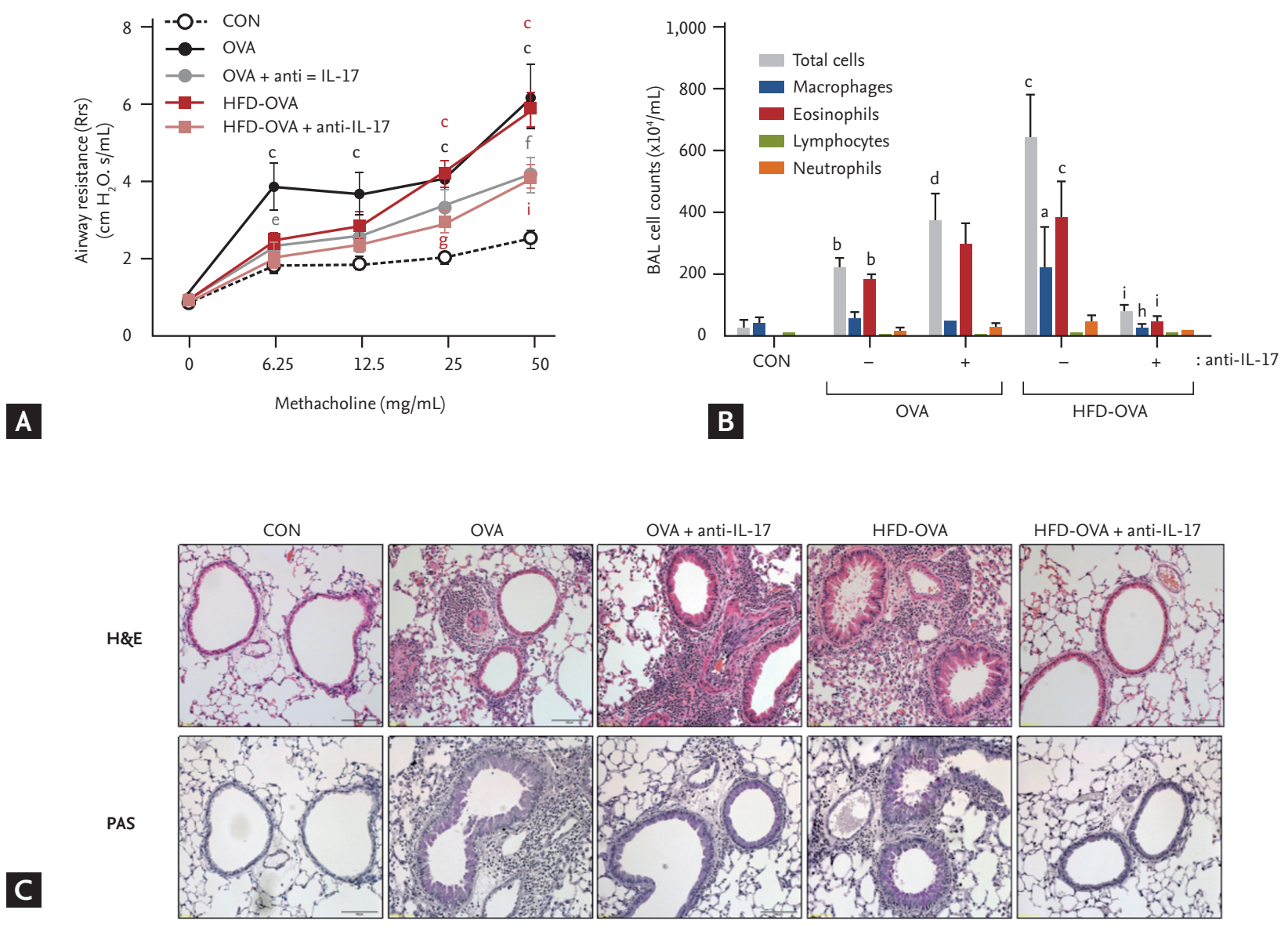

Figure 4. Effects of anti-interleukin 17 (IL-17) antibody treatment on airway hyper-responsiveness (AHR) and allergic airway inflammation in a mouse model of obesity-related asthma. Ovalbumin (OVA) and high-fat diet (HFD)-OVA mice were treated with the anti-IL-17 antibody to deplete IL-17 and were sacrificed 24 hours after the final OVA challenge. (A) AHR and (B) inflammatory cell infiltration in the bronchoalveolar lavage fluid (BALF) were measured. (C) Paraffin-embedded lung tissue sections stained with H\&E and periodic acid-Schiff $(\mathrm{PAS})(\times 200)$. Results are expressed as mean $\pm \mathrm{SEM}$ ( $\mathrm{n}=5$ to 9 per group). CON, control. ${ }^{\mathrm{a}} p<0.05,{ }^{\mathrm{b}} p<0.01,{ }^{\mathrm{c}} p<0.001$ vs. control group, ${ }^{\mathrm{d}} p<0.05,{ }^{\mathrm{e}} p<0.01,{ }^{\mathrm{f}} p<0.001$ vs. OVA group, and ${ }^{\mathrm{g}} p<0.05,{ }^{\mathrm{h}} p<0.01,{ }^{\mathrm{i}} p<$ 0.001 vs. HFD-OVA group

tration as compared to untreated obese asthmatic mice, whereas no difference was detected between anti-IL-17 antibody-treated HFD-OVA mice with and without pravastatin treatment (Fig. 6C). PAS staining revealed that goblet cell proliferation was increased in the HFDOVA group relative to the control, but this was partly reversed by combined pravastatin and anti-IL-17 antibody treatment (Fig. 6C). There was no difference between the HFD-OVA + anti-IL-17 antibody and HFD-OVA + pravastatin + anti-IL-17 antibody groups in terms of airway inflammation. These data suggest that pravastatin and the anti-IL-17 antibody act synergistically to reduce AHR but not airway inflammation in obesity-related asthma.
Pravastatin and anti-IL-17 antibody treatment decreases $T_{H^{2}}$ and $T_{H} 17$ cytokine and changes adipokine levels in obesity-related asthma

We also investigated whether pravastatin and anti-IL-17 antibody treatment affects the expression of $\mathrm{T}_{\mathrm{H}^{2}}$ and $\mathrm{T}_{\mathrm{H}} 17$ cytokines and adipokines in obese asthmatic mice. IL-4, IL-5, IL-13, and IL-17 levels were decreased in the HFD-OVA + pravastatin + anti-IL-17 antibody group as compared to the HFD-OVA group. Moreover, the levels of IL-4, IL-13, and IL-17 were slightly lower in anti-IL-17 antibody-treated HFD-OVA mice versus those without pravastatin treatment (Fig. 7A-7D). Leptin levels in HFDOVA mice were decreased by combined pravastatin and 

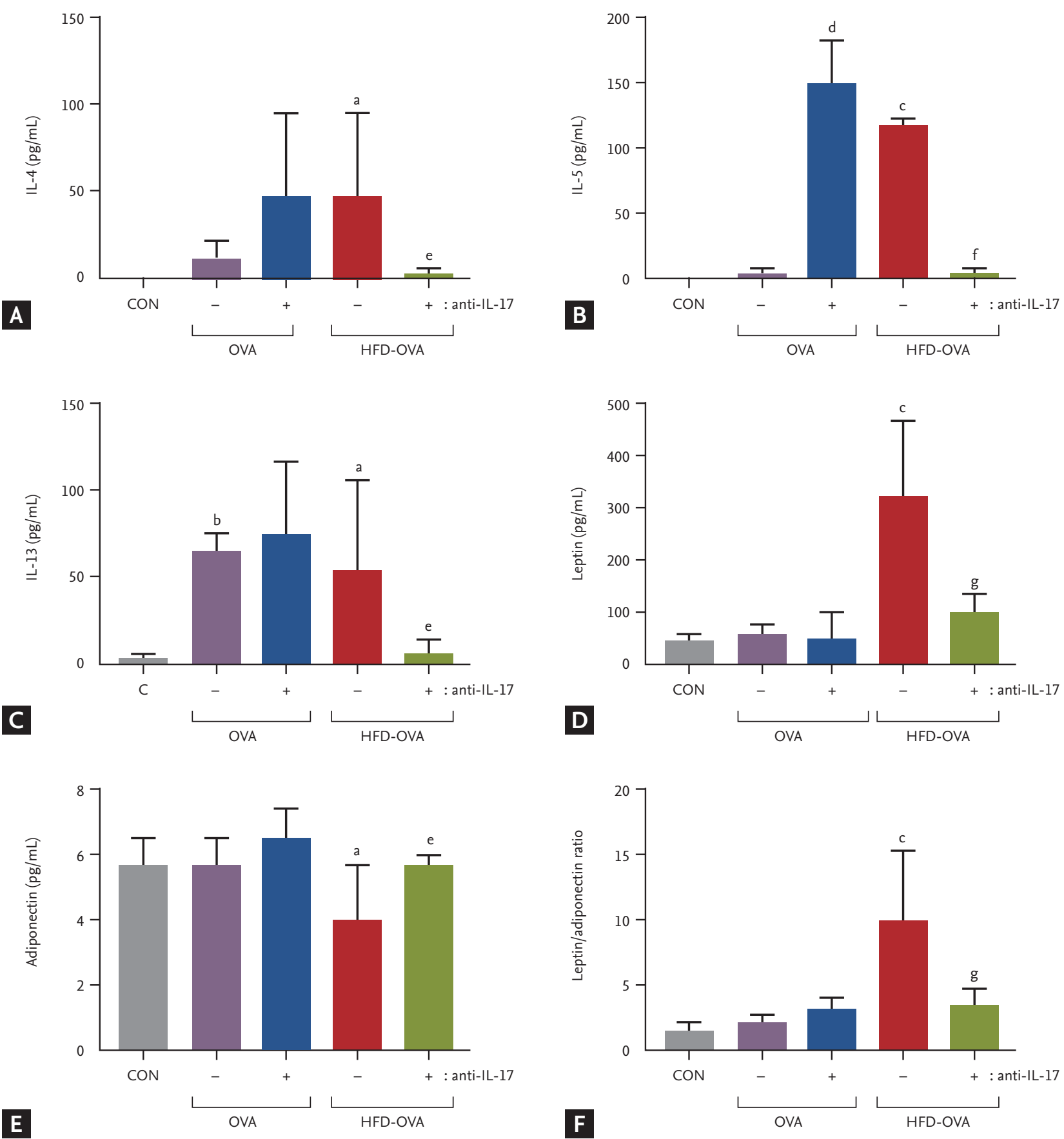

Figure 5. Effects of anti-interleukin 17 (IL-17) antibody treatment on $\mathrm{T}_{\mathrm{H}}$ cytokine and adipokine levels in a mouse model of obesity-related asthma. (A) IL-4, (B) IL-5, (C) IL-13, (D) leptin, and (E) adiponectin levels, and (F) leptin/adiponectin ratio were evaluated in the bronchoalveolar lavage fluid by enzyme-linked immunosorbent assay. Results are expressed as mean \pm SEM (n $=5$ to 9 per group). CON, control; HFD, high-fat diet; OVA, ovalbumin. ${ }^{a} p<0.05,{ }^{b} p<0.01,{ }^{c} p<0.001$ vs. control group, ${ }^{d} p<0.01$ vs. OVA group, and ${ }^{\mathrm{e}} \mathrm{p}<0.05,{ }^{\mathrm{f}} \mathrm{p}<0.01,{ }^{\mathrm{g}} \mathrm{p}<0.001$ vs. HFD-OVA group.

anti-IL-17 antibody treatment, and were slightly lower in HFD-OVA mice treated with both pravastatin and anti-IL-17 antibody as compared to the antibody alone
(Fig. 7 E). Adiponectin levels in HFD-OVA mice were unaffected, whereas the leptin/adiponectin ratio was decreased by combined pravastatin and anti-IL-17 anti- 

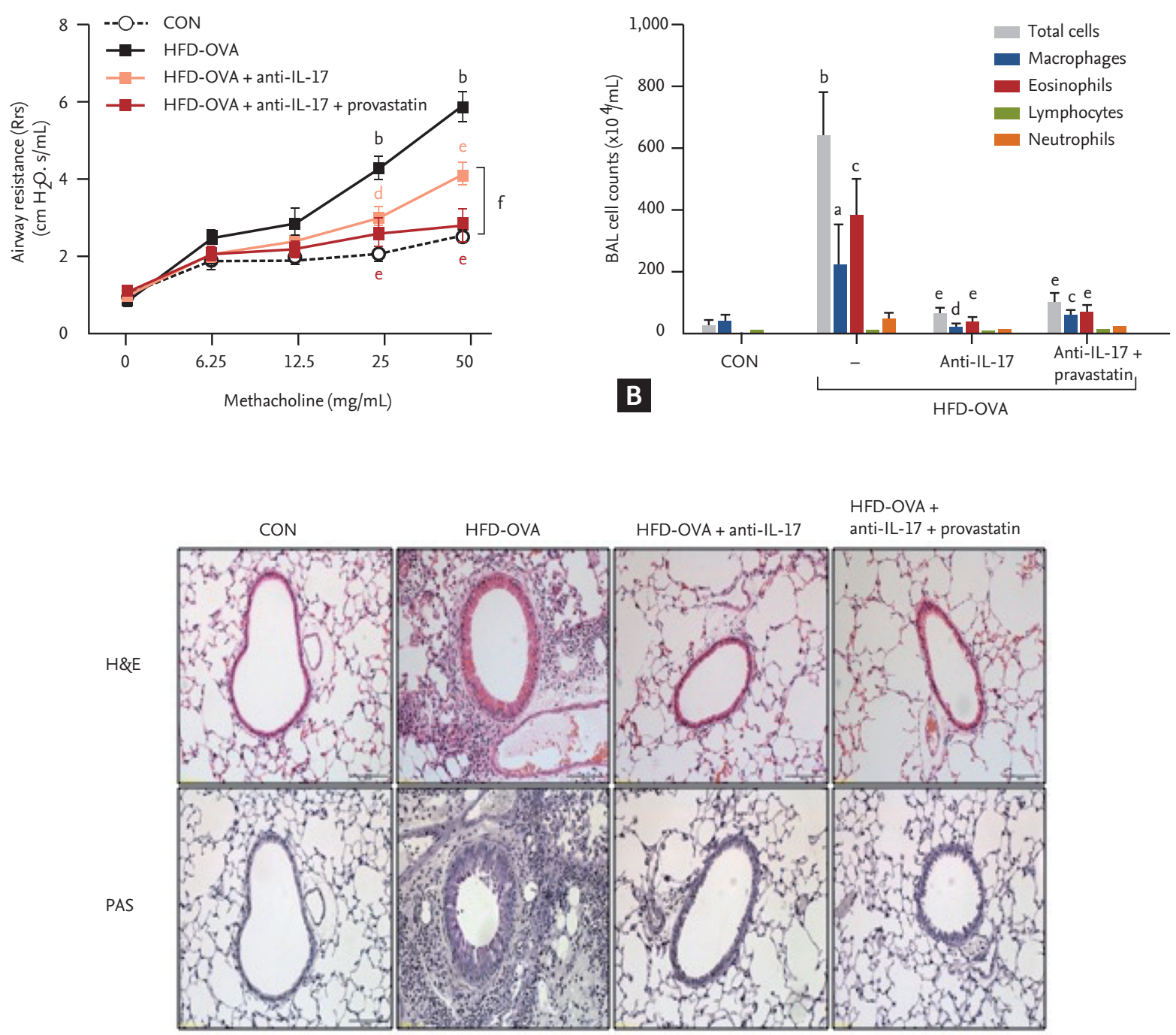

Figure 6. Effects of pravastatin and anti-interleukin 17 (IL-17) antibody on airway hyper-responsiveness (AHR) and allergic airway inflammation in a mouse model of obesity-related asthma. High-fat diet ovalbumin (HFD-OVA) mice were treated with pravastatin + anti-IL-17 antibody. (A) AHR measured as respiratory resistance 24 hours after the final OVA challenge. (B) Inflammatory cell infiltration in the bronchoalveolar lavage fluid (BALF) 24 hours after the final OVA challenge. (C) Paraffin-embedded lung tissue sections stained with H\&E and periodic acid-Schiff $(P A S)(\times 200)$. Results are expressed as mean \pm SEM $(n=$ 5 to 9 per group). CON, control. ${ }^{\mathrm{a}} p<0.05,{ }^{\mathrm{b}} p<0.001$ vs. control group, ${ }^{\mathrm{c}} p<0.05,{ }^{\mathrm{d}} p<0.01,{ }^{\mathrm{e}} p<0.001$ vs. HFD-OVA group, and ${ }^{\mathrm{f}} p$ $<0.05$ vs. HFD-OVA + anti-IL-17 antibody group.

body treatment (Fig. $7 \mathrm{~F}$ ); a less potent effect was observed upon administration of the anti-IL-17 antibody alone (Fig. ${ }_{7} \mathrm{G}$ ). These results demonstrate that pravastatin and the anti-IL-17 antibody act synergistically to suppress $\mathrm{T}_{\mathrm{H}} 2 / \mathrm{T}_{\mathrm{H}} 17$ responses by reducing the leptin/adiponectin ratio in obesity-related asthma.

\section{DISCUSSION}

Obesity is associated with the development and severity of asthma and poor asthma control [3]. It is known to alter lung function directly by altering airway wall thickness and respiratory rate and indirectly by inducing chronic inflammation [4]. Moreover, obese patients with asthma respond less favorably to inhaled cortico- 
A

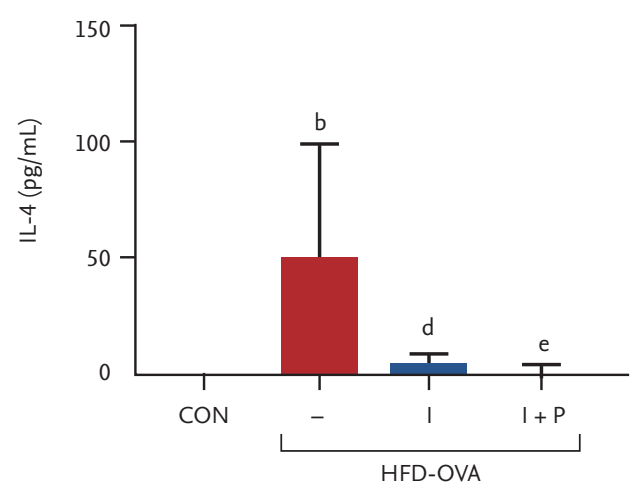

C

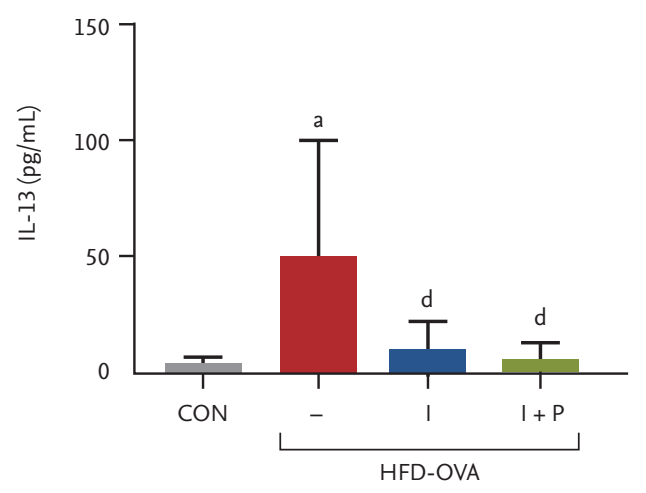

E
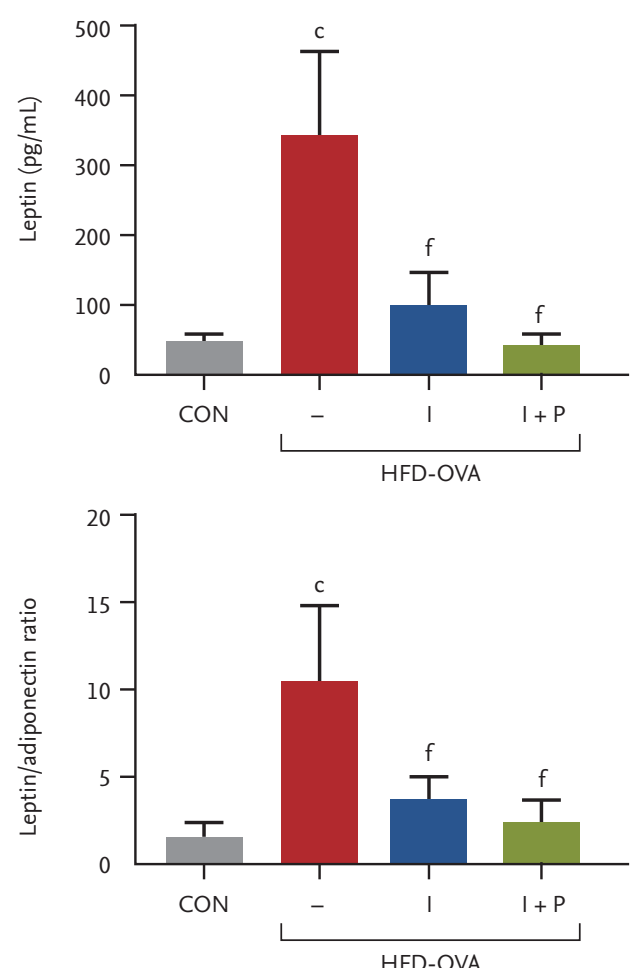

G

HFD-OVA
B

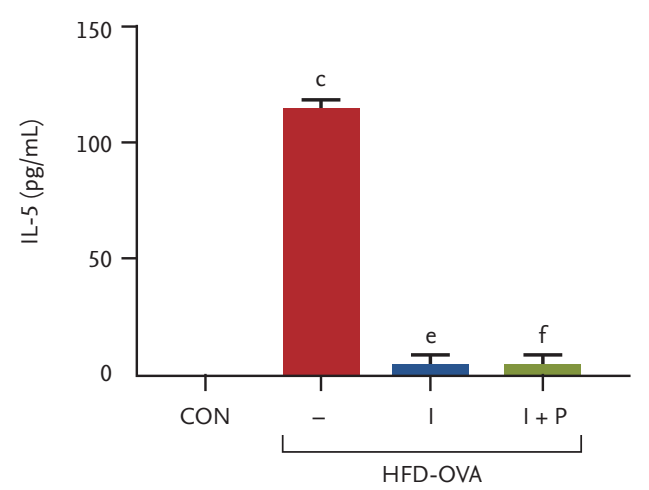

D

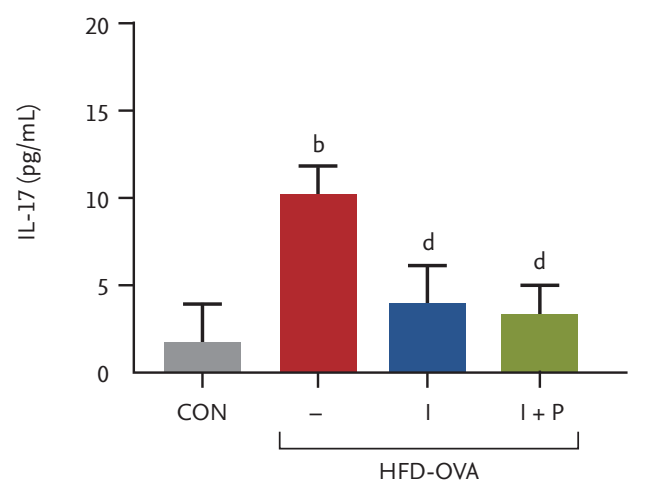

$\mathbf{F}$

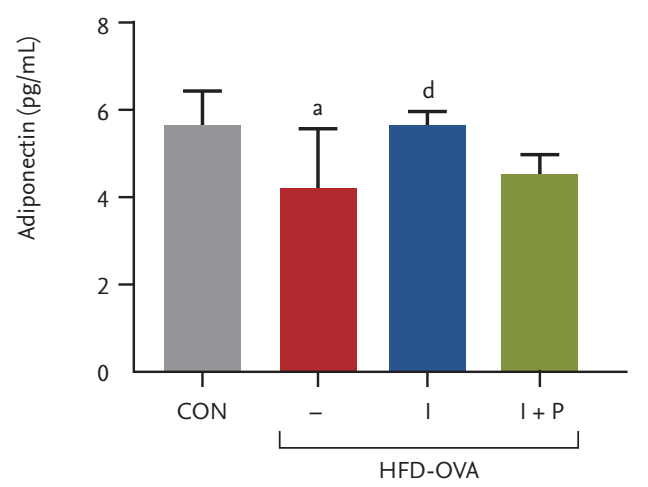

Figure 7. Effects of pravastatin and anti-interleukin 17 (IL-17) antibody treatment on $\mathrm{T}_{\mathrm{H}^{2}}$ and $\mathrm{T}_{\mathrm{H}} 17$ cytokine and adipokine levels in a mouse model of obesity-related asthma. (A) IL-4, (B) IL-5, (C) IL-13, (D) IL-17, (E) leptin, and (F) adiponectin levels, and (G) leptin/adiponectin ratio in the bronchoalveolar lavage fluid were tested by enzyme-linked immunosorbent assay. Results are expressed as mean \pm SEM ( $\mathrm{n}=5$ to 9 per group). CON, control; I, anti-IL-17; P, pravastatin; HFD, high-fat diet; OVA, ovalbumin. ${ }^{\mathrm{a}} p<0.05,{ }^{\mathrm{b}} p<0.01,{ }^{\mathrm{c}} p<0.001$ vs. control group, and ${ }^{\mathrm{d}} p<0.05,{ }^{\mathrm{e}} p<0.01,{ }^{\mathrm{f}} p<0.001$ vs. HFD-OVA group. 
steroids than non-obese asthma patients [8]. The association between obesity and asthma might be explained by the immune-modulatory effect of adipokines secreted by adipose tissue. Recent studies have revealed a link between leptin levels and asthma; high levels of leptin were shown to aggravate airway inflammation and remodeling via the recruitment of eosinophils and neutrophils $[21,22]$. In the present study, we showed that the leptin level in asthmatic mice was increased by the co-occurrence of obesity. Moreover, HFD-OVA mice exhibited greater inflammatory cell infiltration and airway remodeling than OVA mice $(p<0.0001)$ and HFD mice. These data suggest that the link between obesity and asthma involves the immune effects of leptin. A previous study found no associations between leptin and asthma symptoms such as wheezing, airflow obstruction, bronchodilator responsiveness, and airway inflammation [23]. Thus, among allergic inflammatory mediators in obesity, what factors worsen the inflammatory response by increasing leptin?

Obesity-related asthma is characterized by low eosinophil and high airway neutrophil counts. However, recent studies have reported that early-onset asthma patients have higher serum immunoglobulin E levels than their non-obese counterparts [24]. The distribution of eosinophils within different tissues could affect their pathological functions in asthma, particularly in patients with obesity [25]. Thus, obesity-related asthma may be classified as the $\mathrm{T}_{\mathrm{H}^{2}}$ or non- $\mathrm{T}_{\mathrm{H}^{2}}$ type. We measured the levels of $\mathrm{T}_{\mathrm{H}^{2}}$ cytokines (IL-4, IL-5, and IL-13) and $\mathrm{T}_{\mathrm{H}} 17$ cytokines (IL-17) and found that obesity exacerbated airway inflammation in asthma by increasing $\mathrm{T}_{\mathrm{H}^{2}}$ and $\mathrm{T}_{\mathrm{H}} 17$ cytokine levels. Several studies have reported higher $\mathrm{T}_{\mathrm{H}^{2}}$ and $\mathrm{T}_{\mathrm{H}} 17$ cytokine levels in obese asthma as compared to non-obese asthma $[6,12]$.

There is a controversy surrounding the immune functions of obese and non-obese states. Obesity causes a systemic increase in the levels of circulating inflammatory cytokines and leads to immunocyte recruitment to inflamed tissues and leukocyte activation [26]. In the obese state, immunocytes are exposed to higher concentrations of serum leptin; this activates innate immunity, which increases the number of pro-inflammatory $\mathrm{T}_{\mathrm{H}^{1}}$ cells and decreases the number of $\mathrm{T}_{\mathrm{H}^{2}}$ cells $[27,28]$. In addition, chronic obesity induces a switch from M2- to M1-polarized macrophages and stimulates the expres- sion of $\mathrm{T}_{\mathrm{H}^{1}}$-type cytokines [29]. Although a large number of $\mathrm{T}_{\mathrm{H}^{17}}$ cells have been detected in the adipose tissue of lean mice, obesity selectively promotes the proliferation of pro-inflammatory $\mathrm{T}_{\mathrm{H}} 17$ cells [10]. Levels of the proinflammatory cytokine IL-17 are increased in obese patients [30], although this is not correlated with leptin levels. IL-17 has also been implicated in adiponectin production [31]. Interestingly, we found that obese mice showed elevated IL-17 levels relative to control mice, whereas $\mathrm{T}_{\mathrm{H}^{2}}$ cytokines such as IL-4, IL-5, and IL-13 were not increased. This suggests that $\mathrm{T}_{\mathrm{H}} 17$-mediated immunity is critical for inflammatory processes in adipose tissue associated with obesity.

We investigated the role of IL-17 in a mouse model of obesity-induced asthma using a neutralizing anti-IL-17 antibody. IL-17 blockade decreased AHR in both obese and non-obese asthmatic mice. Some studies have suggested that obesity is a risk factor for AHR to methacholine [32,33], although this has been contradicted by other reports [34,35]. Our study showed that AHR was increased in asthmatic mice but was not further aggravated by obesity. This may be explained by the fact that methacholine acts directly on airway smooth muscle receptors to induce airway constriction independent of inflammation. We also found that blocking IL-17 decreased airway inflammation and $\mathrm{T}_{\mathrm{H}^{2}}$ cytokine (IL-4, IL5, and IL-13) levels in obese asthmatic mice. In contrast, IL-17 neutralization increased airway inflammation and $\mathrm{T}_{\mathrm{H}^{2}}$ cytokine levels in non-obese asthmatic mice as compared to controls. Several studies have shown that blocking IL-17 increased airway eosinophilia and decreased AHR in an allergic airway inflammation model, suggesting that a balance between IL-17A and IL-25 is important for modulating allergic lung responses and that allergic asthma is negatively regulated by IL-17 $[36,37]$. Unexpectedly, we found that leptin and adiponectin levels were decreased and increased, respectively, and that the leptin/adiponectin ratio was decreased by anti-IL-17 antibody treatment in obese asthmatic mice, but that these were unaltered in non-obese mice with asthma. Several studies have reported no differences in leptin and adiponectin concentrations between asthma patients and controls, although the leptin/adiponectin ratio was correlated with body mass index in patients [38,39]. Adipokines contribute to the mechanism underlying inflammation and airway constriction. We pro- 
pose that the activation of the IL-17-leptin/adiponectin axis exacerbates asthma symptoms in obese asthma patients compared with non-obese asthma patients. How IL-17 neutralization alleviates AHR in non-obesity-related asthma is not well understood, although it might be independent of airway inflammation. The relationship between AHR and airway inflammation is complex: some reports have demonstrated that blocking of IL-17A reverses the protective effect of AHR by the anti-IL-25 antibody, resulting in the re-expression of $\mathrm{T}_{\mathrm{H}^{2}}$ inflammatory cytokines [36]. On the other hand, IL-17 neutralization has been found to inhibit OVA-induced airway inflammation [40]. Although this controversy has yet to be resolved, our data provide new evidence that the IL17-leptin/adiponectin axis plays a key role in airway inflammation in obesity-related asthma.

The anti-inflammatory effects of statins for the treatment of chronic inflammatory and autoimmune diseases have recently been demonstrated [16]. For example, atorvastatin was found to induce a shift from $\mathrm{T}_{\mathrm{H}^{1}}$ to $\mathrm{T}_{\mathrm{H}^{2}}$ cytokine production [17]. Pravastatin suppressed OVA-induced airway inflammation by reducing $\mathrm{T}_{\mathrm{H}^{2}}$ cytokine levels and IL-17 production [18]. We showed here that the combination of the anti-IL-17 antibody and pravastatin synergistically reduced AHR in obese asthmatic mice while having no effect on IL-4, IL-5, or IL-13 expression. We also compared the HFD-OVA + pravastatin, HFD-OVA + anti-IL-17 antibody, and HFD-OVA + pravastatin + anti-IL-17 antibody groups. Pravastatin treatment alone also reduced eosinophilic airway inflammation and AHR and increased the levels of $\mathrm{T}_{\mathrm{H}^{2}}$ cytokines in asthma with and without obesity. However, there was no difference between the HFD-OVA + pravastatin and HFD-OVA + anti-IL-17 antibody groups in terms of AHR and airway inflammation (data not shown). These data suggested that pravastatin acts upstream of the "IL-17-leptin/adiponectin axis" and has a synergistic effect with the anti-IL-17 antibody on AHR. However, it does not act in a parallel pathway on airway inflammation. Pravastatin attenuates allergic inflammation in a mouse model of obesity-related asthma, which might be related with other pathways. Further studies using various cytokine agonists in pravastatin-treated obesity-associated asthma models are required to investigate the comprehensive details.

In summary, we demonstrated that anti-IL-17 anti- body treatment attenuated AHR and airway inflammation and increased $\mathrm{T}_{\mathrm{H}^{2}} / \mathrm{T}_{\mathrm{H}^{17}}$ cytokine levels by suppressing the IL-17-leptin/adiponectin axis in obese asthmatic mice. This is the first report of an association between this axis and obesity-related asthma. Our findings might offer clues for developing the new pharmaceuticals for treating obesity-related asthma.

\section{KEY MESSAGE}

1. Obesity exacerbates allergic airway inflammation in a mouse asthma model.

2. The interleukin 17-leptin/adiponectin axis plays a key role in airway inflammation in obesity-related asthma.

\section{Conflict of interest}

No potential conflict of interest relevant to this article was reported.

\section{Acknowledgments}

This research was supported by the National Research Foundation of Korea (NRF) grant funded by the Ministry of Science, ICT \& Future Planning (No. 2016RiDiA1Ao-2937276).

\section{REFERENCES}

1. Bastard JP, Maachi M, Lagathu C, et al. Recent advances in the relationship between obesity, inflammation, and insulin resistance. Eur Cytokine Netw 2006;17:4-12.

2. Olin JT, Wechsler ME. Asthma: pathogenesis and novel drugs for treatment. BMJ 2014;349:g5517.

3. Muc M, Mota-Pinto A, Padez C. Association between obesity and asthma: epidemiology, pathophysiology and clinical profile. Nutr Res Rev 2016;29:194-201.

4. Delgado J, Barranco P, Quirce S. Obesity and asthma. J Investig Allergol Clin Immunol 2008;18:420-425.

5. King GG, Brown NJ, Diba C, et al. The effects of body weight on airway calibre. Eur Respir J 2005;25:896-901.

6. Calixto MC, Lintomen L, Schenka A, Saad MJ, Zanesco A, Antunes E. Obesity enhances eosinophilic inflammation in a murine model of allergic asthma. Br J Pharmacol 2010;159:617-625. 
7. Lintomen L, Calixto MC, Schenka A, Antunes E. Allergen-induced bone marrow eosinophilopoiesis and airways eosinophilic inflammation in leptin-deficient ob/ob mice. Obesity (Silver Spring) 2012;20:1959-1965.

8. Baffi CW, Winnica DE, Holguin F. Asthma and obesity: mechanisms and clinical implications. Asthma Res Pract 2015;1:1.

9. Wenzel SE. Asthma phenotypes: the evolution from clinical to molecular approaches. Nat Med 2012;18:716-725.

10. Endo Y, Yokote K, Nakayama T. The obesity-related pathology and Th17 cells. Cell Mol Life Sci 2017;74:1231-1245.

11. Leiria LO, Martins MA, Saad MJ. Obesity and asthma: beyond $\mathrm{T}(\mathrm{H}) 2$ inflammation. Metabolism 2015;64:172-181.

12. Kim HY, Lee HJ, Chang YJ, et al. Interleukin-17-producing innate lymphoid cells and the NLRP3 inflammasome facilitate obesity-associated airway hyperreactivity. Nat Med 2014;20:54-61.

13. Kudo M, Melton AC, Chen C, et al. IL-17A produced by $\alpha \beta$ $\mathrm{T}$ cells drives airway hyper-responsiveness in mice and enhances mouse and human airway smooth muscle contraction. Nat Med 2012;18:547-554.

14. Agache I, Ciobanu C, Agache C, Anghel M. Increased serum IL-17 is an independent risk factor for severe asthma. Respir Med 2010;104:1131-1137.

15. Wang CY, Liu PY, Liao JK. Pleiotropic effects of statin therapy: molecular mechanisms and clinical results. Trends Mol Med 2008;14:37-44.

16. Zhang X, Markovic-Plese S. Statins' immunomodulatory potential against Th17 cell-mediated autoimmune response. Immunol Res 2008;41:165-174.

17. Youssef S, Stuve O, Patarroyo JC, et al. The HMG-CoA reductase inhibitor, atorvastatin, promotes a Th2 bias and reverses paralysis in central nervous system autoimmune disease. Nature 2002;420:78-84.

18. Imamura M, Okunishi $\mathrm{K}$, Ohtsu $\mathrm{H}$, et al. Pravastatin attenuates allergic airway inflammation by suppressing antigen sensitisation, interleukin 17 production and antigen presentation in the lung. Thorax 2009;64:44-49.

19. Celedon JC, Kolls JK. An innate link between obesity and asthma. Nat Med 2014;20:19-20.

20. Mraz M, Haluzik M. The role of adipose tissue immune cells in obesity and low-grade inflammation. J Endocrinol 2014;222:R113-R127.

21. Zarkesh-Esfahani H, Pockley AG, Wu Z, Hellewell PG, Weetman AP, Ross RJ. Leptin indirectly activates human neutrophils via induction of TNF-alpha. J Immunol
2004;172:1809-1814.

22. Kato H, Ueki S, Kamada R, et al. Leptin has a priming effect on eotaxin-induced human eosinophil chemotaxis. Int Arch Allergy Immunol 2011;155:335-344.

23. McLachlan CR, Poulton R, Car G, et al. Adiposity, asthma, and airway inflammation. J Allergy Clin Immunol 2007;119:634-639.

24. Holguin F, Bleecker ER, Busse WW, et al. Obesity and asthma: an association modified by age of asthma onset. J Allergy Clin Immunol 2011;127:1486-1493.

25. Wu D, Molofsky AB, Liang HE, et al. Eosinophils sustain adipose alternatively activated macrophages associated with glucose homeostasis. Science 2011;332:243-247.

26. Ignacio RM, Kim CS, Kim SK. Immunological profiling of obesity. J Lifestyle Med 2014;4:1-7.

27. Lord G. Role of leptin in immunology. Nutr Rev 2002;60(10 Pt 2):S35-S38.

28. Brestoff JR, Kim BS, Saenz SA, et al. Group 2 innate lymphoid cells promote beiging of white adipose tissue and limit obesity. Nature 2015;519:242-246.

29. Lumeng CN, Bodzin JL, Saltiel AR. Obesity induces a phenotypic switch in adipose tissue macrophage polarization. J Clin Invest 2007;117:175-184.

30. Sumarac-Dumanovic M, Stevanovic D, Ljubic A, et al. Increased activity of interleukin-23/interleukin-17 proinflammatory axis in obese women. Int J Obes (Lond) 2009;33:151-156.

31. Zuniga LA, Shen WJ, Joyce-Shaikh B, et al. IL-17 regulates adipogenesis, glucose homeostasis, and obesity. J Immunol 2010;185:6947-6959.

32. Litonjua AA, Gold DR. Asthma and obesity: common early-life influences in the inception of disease. J Allergy Clin Immunol 2008;121:1075-1084.

33. Sood A, Ford ES, Camargo CA Jr. Association between leptin and asthma in adults. Thorax 2006;61:300-305.

34. Schachter LM, Salome CM, Peat JK, Woolcock AJ. Obesity is a risk for asthma and wheeze but not airway hyperresponsiveness. Thorax 2001;56:4-8.

35. Bustos P, Amigo H, Oyarzun M, Rona RJ. Is there a causal relation between obesity and asthma? Evidence from Chile. Int J Obes (Lond) 2005;29:804-809.

36. Barlow JL, Flynn RJ, Ballantyne SJ, McKenzie AN. Reciprocal expression of IL-25 and IL-17A is important for allergic airways hyperreactivity. Clin Exp Allergy 2011;41:1447-1455.

37. Schnyder-Candrian S, Togbe D, Couillin I, et al. Interleu- 
kin-17 is a negative regulator of established allergic asthma. J Exp Med 2006;203:2715-2725.

38. Jartti T, Saarikoski L, Jartti L, et al. Obesity, adipokines and asthma. Allergy 2009;64:770-777.

39. Jang AS, Kim TH, Park JS, et al. Association of serum leptin and adiponectin with obesity in asthmatics. J Asthma 2009;46:59-63.

40. Song C, Luo L, Lei Z, et al. IL-17-producing alveolar macrophages mediate allergic lung inflammation related to asthma. J Immunol 2008;181:6117-6124. 